\title{
Correspondance du président de Brosses et de l'abbé marquis Niccolini, sous la direction de John ROGISTER et Mireille GILLE
}

\author{
Maria Immacolata Spagna
}

\section{(2) OpenEdition}

\section{Journals}

\section{Edizione digitale}

URL: https://journals.openedition.org/studifrancesi/11571

DOI: 10.4000/studifrancesi. 11571

ISSN: 2427-5856

\section{Editore}

Rosenberg \& Sellier

\section{Edizione cartacea}

Data di pubblicazione: 1 avril 2018

Paginazione: 129-130

ISSN: 0039-2944

\section{Notizia bibliografica digitale}

Maria Immacolata Spagna, «Correspondance du président de Brosses et de l'abbé marquis Niccolini, sous la direction de John rogISTER et Mireille GILLE», Studi Francesi [Online], 184 (LXII | I) | 2018, online dal 02 juillet 2018, consultato il 15 novembre 2021. URL: http://journals.openedition.org/studifrancesi/11571 ; DOI: https://doi.org/10.4000/studifrancesi. 11571

Questo documento è stato generato automaticamente il 15 novembre 2021.

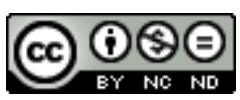

Studi Francesi è distribuita con Licenza Creative Commons Attribuzione - Non commerciale - Non opere derivate 4.0 Internazionale. 


\title{
Correspondance du président de Brosses et de l'abbé marquis Niccolini, sous la direction de John ROGISTER et Mireille GILLE
}

\author{
Maria Immacolata Spagna
}

\section{NOTIZIA}

Correspondance du président de Brosses et de l'abbé marquis Niccolini, sous la direction de John ROGISTER et Mireille GILLE, Oxford, Voltaire Foundation, 2016, «Oxford University Studies in the Enlightenment» 12, 289 pp.

1 Frutto di anni di ricerche, il volume raccoglie la corrispondenza tra il presidente de Brosses e l'abate Niccolini. Si tratta di quarantotto lettere, in gran parte inedite. Qui presentate in ordine cronologico, esse coprono un periodo compreso fra l's luglio del 1740 e il 14 ottobre 1770 .

2 Questa corrispondenza è innanzitutto un lungo dialogo epistolare tra due eruditi: Charles de Brosses, conte di Tourney e di Motfalcon, parlamentare implicato nelle controversie politiche del regno di Luigi XV, autore di numerose opere, ben noto agli storici e agli studiosi della letteratura di viaggio del xviII secolo, e Antonio Niccolini, nobile fiorentino, meno conosciuto, marchese di Ponsacco e di Camugliano, attratto da una carriera diplomatica al servizio della Santa Sede ostacolata dal suo spirito libero e dalla sua franchezza, che gli procurarono non pochi nemici nella curia. I due si incontrarono per la prima volta in occasione del viaggio di de Brosses in Italia, a Firenze, nel 1739, e da allora restarono legati da un'amicizia che si espresse in un fitto scambio epistolare, animato dai loro interessi comuni e dalla curiosità per le novità letterarie, storiche e scientifiche, che durerà trent'anni. 
3 Ricche di citazioni di Orazio e di Virgilio, ma anche di Cicerone, Molière, Ariosto, Giovenale, Spinoza, Dante, Ovidio, le lettere evocano tantissimi autori: Newton, Montesquieu, Voltaire, Hume, Buffon, Wolff, ecc.; attorno ad essi, appaiono libri e raccolte che estendono il campo delle conoscenze umane. Le lettere, grazie anche alle puntuali note biografiche relative ai nomi citati, permettono di approfondire la conoscenza di personalità dell'epoca come, ad esempio, l'amico di Niccolini, Antonio Francesco Gori, archeologo ed erudita cui si devono i primi sei volumi del Museum Florentinum, impresa nella cui pubblicazione l'abate fiorentino ha svolto un ruolo di primo piano (lettera III nota 24); il matematico Charles Marie de La Condamine, l'astronomo Louis Godin, il matematico, astronomo e idrografo Pierre Boguer, tutti nomi legati alla spedizione in Perù che aveva l'obiettivo di determinare con precisione la latitudine (lettera IX note 58, 59, 60); e ancora Giovanni Giacomo Marinoni, cartografo e astronomo che aveva perfezionato uno strumento per calcolare le superfici (lettera XII nota 99).

4 La corrispondenza è altresì una lunga conversazione tra due amici che raccontano avvenimenti familiari e si abbandonano a confidenze personali, come capita nella lettera XLV, dove Niccolini parla della morte di due suoi nipoti, della sua malattia e dei processi che lo hanno tormentato, o nella lettera XLVI, in cui de Brosses esprime il suo sconforto per la morte della moglie, e successivamente, nella lettera XLVIII, quando informa l'abate della morte di suo figlio, del matrimonio della figlia e del suo nuovo matrimonio con Jeanne-Marie Le Gouz de Saint-Seine. Niccolini rende puntualmente partecipe de Brosses delle impressioni sul suo lungo viaggio (dal 1745 al 1748), nel corso del quale incontrerà diversi eruditi.

5 Trattando temi che spaziano dalle questioni politiche alle ultime scoperte scientifiche, dalla filosofia alla storia e alla letteratura, la corrispondenza tra il presidente de Brosses e l'abate Niccolini è di grande interesse storico, politico, letterario e scientifico, e contribuisce alla storia degli scambi culturali tra i membri della "République des lettres", permettendo di cogliere meglio la mentalità di una certa élite. Dal punto di vista prettamente letterario, fornisce chiarimenti e precisazioni sulla genesi delle famose Lettres familières sur l'Italie di de Brosses. Inoltre, dal momento che i due uomini hanno vissuto un periodo storicamente e politicamente rilevante, di cui si trova l'eco nella loro corrispondenza - non mancano riflessioni sul ruolo della Francia alla vigilia del trattato di Aquisgrana, sulla dominazione marittima e coloniale inglese nel corso della Guerra dei Sette Anni, sulla distruzione del potere dell'ordine dei gesuiti, sul declino politico inesorabile del Granducato di Toscana -, le lettere costituiscono un'occasione per approfondire la conoscenza degli avvenimenti significativi dell'epoca e delle ragioni alla base della politica interna ed estera degli Stati europei. Niccolini, in particolare, si rivela un fine giudice della scena diplomatica e dimostra una grande perspicacia sulle questioni di politica estera.

6 Oltre all'innegabile valore letterario e storico di queste missive, non va trascurato il loro interesse filologico e linguistico. Coprendo un periodo di trent'anni, queste lettere, spontanee, non destinate ad essere pubblicate, contenenti errori non imputabili a segretari o copisti bensì agli autori stessi, testimoniano dell'evoluzione dell'ortografia $\mathrm{e}$ costituiscono un importante documento da sfruttare per molteplici riflessioni. Mossi dalla volontà di rispettare l'autenticità del testo, i curatori della presente edizione hanno scelto di offrirne una trascrizione quanto più fedele possibile, privilegiando la leggibilità e la comprensione del manoscritto, in modo da ottenere un corpus fruibile 
per ulteriori studi. Con questo obiettivo, riportano l'ortografia delle lettere dell'abate fiorentino che molto spesso trascrive foneticamente la sua pronuncia italianizzata del francese, disseminando errori di accentuazione, oltre a quelli sintattici tipici degli italofoni.

7 Ad arricchire ulteriormente il volume contribuiscono le lettere indirizzate a uno o all'altro dei due amici, raccolte in appendice (pp. 211-266), come le tre lettere di Montesquieu a Niccolini, le quattordici di de La Curne de Sainte-Palaye, le due lettere di Monsignore Cerati o le lettere del conte e della contessa Lorenzi a de Brosses.

Chiudono il volume una corposa bibliografia (pp. 267-277) e un indice (pp. 279-289) che elenca tutti i nomi delle personalità evocate nella corrispondenza.

9 Attraverso questo denso scambio epistolare, il volume proposto da John Register e Mireille Gille rivela il pensiero di due uomini di alta cultura, testimoni e attori dello sviluppo intellettuale delle élites europee del xviII secolo; rappresenta pertanto un prezioso documento per l'approfondimento della conoscenza di un secolo attraversato da importanti sconvolgimenti culturali, sociali, filosofici e scentifici. 